\title{
Mediastinoscopy in Assessing Mediastinal Lymphadenopathy and Lung Disease
}

\author{
Iskander Al-Githmi \\ Department of Surgery, Division of Cardiothoracic Surgery, King Abdulaziz University Hospital, Jeddah, KSA \\ Email: algithmi@hotmail.com
}

How to cite this paper: Al-Githmi, I. (2017) Mediastinoscopy in Assessing Mediastinal Lymphadenopathy and Lung Disease. Open Journal of Thoracic Surgery, 7, 55-61.

https://doi.org/10.4236/ojts.2017.74008

Received: October 17, 2017

Accepted: December 2, 2017

Published: December 5, 2017

Copyright $\odot 2017$ by author and Scientific Research Publishing Inc. This work is licensed under the Creative Commons Attribution-NonCommercial International License (CC BY-NC 4.0).

http://creativecommons.org/licenses/by-nc/4.0/

\begin{abstract}
Background: Mediastinoscopy is the gold standard procedure for the pathological staging and diagnosis of mediastinal diseases. The aim of the study is to describe the significance of anterior cervical Mediastinoscopy in evaluating patients with mediastinal lymphadenopathy and lung parenchymal disease. Material and Methods: From January 2014 until June 2017 we conducted a retrospective study of 52 consecutive patients with mediastinal lymphadenopathy and lung parenchymal disease who underwent anterior cervical Mediastinoscopy. The main indications for cervical Mediastinoscopy were isolated mediastinal lymphadenopathy, undetermined lung mass with mediastinal lymphadenopathy, and the clinical staging of patients with lung cancer. Results: The study subjects consisted of 37 men and 15 women, aged 13 to 87 (mean age 50.8). Eight patients had mediastinoscopy for staging lung cancer, 27 patients had mediastinoscopy to diagnose isolated mediastinal lymphadenopathy, and 17 patients had mediastinoscopy for an undetermined lung parenchymal mass. Among the patients with isolated mediastinal lymphadenopathy, sarcoidosis was diagnosed in 6 , tuberculosis in 9 , reactive lymph nodes in 4, metastasis in 5 and lymphoma in 3. Lymph node metastasis (N2, N3) was found in 5 patients with lung cancer. There was no surgical-related mortality or morbidity. Conclusions: Mediastinoscopy is a safe and reliable procedure in diagnosing mediastinal diseases and is still the first choice among the investigative modalities in the clinical staging of bronchogenic carcinoma.
\end{abstract}

\section{Keywords}

Mediastinoscopy, Mediastinum, Lymphadenopathy, Lung, Cancer

\section{Introduction}

Mediastinoscopy was introduced by Carlens in 1959 [1] and has since become an 
integral part of evaluating mediastinal diseases and of the clinical staging of patients with lung cancer. The efficacy of cervical mediastinoscopy for the clinical staging of bronchogenic carcinoma is well established, with a sensitivity of more than $90 \%$ and specificity of $100 \%$ [2] [3] [4]. Non-invasive approaches, such as computed tomography (CT) integrated with positron emission tomography (PET), have caused thoracic surgeons to doubt the importance of cervical mediastinoscopy in the clinical diagnosis of mediastinal masses and the staging of lung cancer. Mediastinoscopy is critical in effectively diagnosing or treating medical conditions based on evidence-based practice since it enables the healthcare providers to view the contents of mediastinum or obtain a biopsy. It helps the doctors to explore the chest and gather key information that can help understand critical conditions as well as ensure effective treatments adopted. However, a tissue sample must be obtained for pathologic diagnosis and treatment strategies.

\section{Material and Methods}

This study is a retrospective, chart-computer database review of all mediastinoscopies performed between January 2014 and June 2017 at King Abdulaziz University Hospital in Jeddah, Saudi Arabia. During the study period, 52 patients underwent mediastinoscopy to assess mediastinal lymphadenopathy and lung parenchymal disease; 25 of these were suspected or confirmed to have lung cancer. Eight patients had a high clinical suspicion of mediastinal metastasis based on lymph node size of more than $1 \mathrm{~cm}$ in short-axis diameter based on a CT scan. Cervical mediastinoscopy was performed for each of these patients and was retrospectively analyzed. Final bronchoscopy, pathology and cytology results were documented.

\section{Results}

A total of 52 patients underwent cervical mediastinoscopy, 37 (71\%) men and 15 (29\%) women with a mean age of 50.8 (range 13 to 87 years). Of these, 27 (54\%) underwent mediastinoscopy for isolated mediastinal lymphadenopathy, 8 (15\%) underwent mediastinoscopy for known lung cancer and 17 (32\%) underwent mediastinoscopy for an undetermined lung mass or the presence of a parenchymal lung lesion (Table 1). Of the 8 (15\%) patients, mediastinoscopy revealed lymph node metastasis in 5 (62\%) patients. Of the 27 (54\%) patients for whom mediastinoscopy was performed to evaluate mediastinal lymphadenopathy in the absence of lung parenchymal diseases: 6 were found to have sarcoidosis, 9 were found to have tuberculosis, 4 were found to have reactive lymph nodes, 5 were found to have metastasis and 3 were found to have lymphoma. Of the 17 patients with an undetermined lung mass, 9 were found to have metastasis, 2 were found to have tuberculosis, 1 was found to have sarcoidosis, 1 was found to have lymphoma and 4 were found to have reactive lymph nodes (Table 2). The patients for whom mediastinoscopy revealed no evidence of N2 disease under- 
Table 1. Patient characteristics.

\begin{tabular}{lc}
\hline Patient characteristics & $\mathbf{N}=\mathbf{5 2}$ \\
\hline Age, mean (years) & 50.8 \\
Gender, no. (\%) & $37(71)$ \\
Male & $15(29)$ \\
Female & \\
Preoperative diagnosis, no. (\%) & $8(15)$ \\
Lung Cancer & $27(54)$ \\
Isolated lymphadenopathy & $17(32)$ \\
Undetermined parenchymal mass & \\
\hline
\end{tabular}

Table 2. Histologic diagnosis crosstabulation.

\begin{tabular}{ccccccc}
\hline Preoperative diagnosis & \multicolumn{5}{c}{ Histologic diagnosis } \\
\hline & Total & Metastasis & Tuberculosis & Sarcoidosis & Lymphoma & Reactive \\
\hline Lung cancer & 8 & 5 & 0 & 0 & 0 & 3 \\
Isolated Lymphadenopathy & 27 & 5 & 9 & 6 & 3 & 4 \\
Undertermined lung mass & 17 & 9 & 2 & 1 & 7 & 4 \\
Total & 52 & 19 & 11 & 7 & 11
\end{tabular}

went thoracotomy and resection, whereas patients with the N2 disease were referred for a neoadjuvant protocol.

We had no surgical-related mortality or morbidity in our series. In our study, the sensitivity, specificity and negative predictive value of cervical mediastinoscopy in patients with primary lung cancer were found to be $100 \%, 73 \%$, and $100 \%$, respectively. In patients with isolated mediastinal lymphadenopathy, we found the cervical mediastinoscopy sensitivity, specificity, and negative predictive value was $100 \%$.

\section{Discussion}

Cervical mediastinoscopy is the gold standard method for the diagnosis and staging of mediastinal pathology. Imaging with $\mathrm{CT}$ and PET is neither sensitive nor specific enough to detect nodal metastasis. Several studies have compared CT to mediastinoscopy in the accurate staging of bronchogenic carcinoma [5] [6] [7]. A mediastinum that appears normal upon CT may harbor metastatic disease in $13 \%$ of cases. It is well known that a positive CT result predicts actual metastatic involvement in half of the patients, and these patients require mediastinoscopy to confirm or rule out micrometastasis [8]. Mediastinoscopy has proved to be a safe procedure with an extremely low complication rate. Even with the development of advanced imaging studies, it still has high sensitivity and specificity. In a study of 181 patients, Rodriguez et al. recorded a sensitivity of $93.6 \%$ and a specificity of $100 \%$ [9]. Porte and co-workers performed 400 me- 
diastinoscopies with a sensitivity of $96 \%$ and a specificity of $100 \%$.

The advent of various non-invasive or minimally invasive techniques for evaluating the mediastinum has led to various studies, some of which have compared endobronchial ultrasound transbronchial needle aspiration (EBUS-TBNA) to mediastinoscopy. Kasufuku and colleagues undertook a prospective controlled trial involving 159 patients and compared EBUS-TBNA with mediastinoscopy as staging modalities for assessing mediastinal lymph nodes in patients with lung cancer; they indicated the superiority of mediastinoscopy for sampling lymph node stations $2 \mathrm{R}$ and $2 \mathrm{~L}$, although there was no significant difference in determining the true pathologic mediastinal lymph node stage. The authors reported a negative predictive value of $91 \%$ and $90 \%, 2 \mathrm{R}$ and $2 \mathrm{~L}$ respectively [10]. Recently, the functional PET has been used in the staging of bronchial carcinoma. PET in addition to CT has been found to be superior to CT scans alone in evaluating mediastinal lymph nodes in non-small lung cancer [11] [12]. Although PET-CT has high sensitivity, about 10\% - 15\% of mediastinal lymph node metastasis from lung cancer will not be detected by PET-CT scans [13] [14].

Therefore, PET-CT scans do not reduce the need for cervical mediastinoscopy in detecting nodal disease [15]. Our study supports the use of mediastinoscopy in the preoperative staging of lung cancer. We found mediastinoscopy had a sensitivity of $100 \%$ in the diagnosis of N2 disease. Our policy was to do mediastinoscopy even in the absence of enlarged mediastinal lymph nodes upon CT scans. We routinely sample prevascular lymph nodes (stations 5 and 6) in patients with left upper lobe cancer and perform left anterior mediastinotomy (Chamberlain procedure) before resection. We do not perform lung resection on patients with N2 proven by mediastinoscopy [16]. In their study, Cybulsky et al. reported the five-year survival rate of patients with the resected $\mathrm{N} 2$ disease to be $6.6 \%$. We believe the resection of $\mathrm{N} 2$ disease is inappropriate and that mediastinoscopy has saved patients from unnecessary major resection [17]. Other methods for obtaining histopathology specimen of enlarged mediastinal lymph nodes include Video-assisted thoracoscopic surgery which gives direct visualization and access to remove aortopulmonary and inferior pulmonary ligament lymph node zones. But this procedure is limited to an assessment of only one side of the mediastinum. Mediastinoscopy promotes the chances of getting more accurate information on the patients conditions compared to other assessment methods of diagnosis.

We believe that cervical mediastinoscopy remains the gold standard minimally invasive procedure with high predictive value and a low incidence of complications [18]. But mediastinoscopy should not be taken simply and lightly as major complications were reported in the literature. Intraoperative bleeding, postoperative pneumothorax, recurrent laryngeal nerve injury, tracheal laceration, esophageal injury and wound infection have been reported. In experienced hands, cervical mediastinoscopy has no mortality and has morbidity as low as 
$2.3 \%$ [19]. In our experience, we did not observe any major complications related to mediastinoscopy. Mass designs has been made to establish the value of mediastinoscopy in assessing mediastinal diseases for many years with the key motivation being the effectiveness of the method compared to the new non-invasive approaches adopted. The key disadvantage with the condition is based on the idea that it can cause adverse effects which can be fatal for the patient during the surgery. The method offers a chance to get more accurate results while ensuring staging mediastinal lymph node involvement.

\section{Conclusion}

In conclusion, it is mandatory to obtain a diagnosis of mediastinal nodal status with less or noninvasive methods such as EBUS-TBNA and PET/CT scan. However, these procedures are not adequately sufficient to provide adequate diagnostic yield. Finally, Mediastinoscopy is safe, effective and can provide an accurate evaluation of thoracic disease and mediastinal lymph nodes status in the staging of lung cancer. The value of mediastinoscopy is profound in healthcare despite the introduction of new non-invasive methods since it offers healthcare providers a clear perspective on the patient's condition thus promoting positive patient outcomes. However follow-up is critical to ensure the patients' side effects are effectively recorded since they vary based on their medical conditions.

\section{References}

[1] Carlens, E. (1959) Mediastinoscopy: A Method for Inspection and Tissue Biopsy in the Superior Mediastinum. Diseases of the Chest, 36, 343-349. https://doi.org/10.1378/chest.36.4.343

[2] Van Schil, P.E.Y., Van Hee, R.H.G.G. and Schools, E.L.G. (1989) The Value of Mediastinoscopy in Preoperative Staging of Bronchogenic Carcinoma. The Journal of Thoracic and Cardiovascular Surgery, 97, 240-244.

[3] Luke, W.P., Pearson, F.G., Todd, T.P.U., et al. (1986) Prospective Evaluation of Mediastinoscopy for Assessment of Carcinoma of the Lung. The Journal of Thoracic and Cardiovascular Surgery, 91, 53-56.

[4] Gdeedo, A., Van Schill, P., Corthouts, B., et al. (1997) Prospective Evaluation of Computed Tomography and Mediastinoscopy in Mediastinal Lymph Node Staging European Respiratory Journal, 10, 1547-1551.

[5] Funatsu, T., Matsubara, Y., Hatakenaka, R., et al. (1992) The Role of Mediastinoscopic Biopsy in Preoperative Assessment of Lung Cancer. The Journal of Thoracic and Cardiovascular Surgery, 104, 1688-1695.

[6] De Leyn, P., Vansteenkiste, J., Cuypers, P., et al. (1997) Role of Cervical Mediastinoscopy in Staging of Non-Small Cell Lung Cancer without Enlarged Mediastinal Lymph Nodes on CT Scan. European Journal of Cardio-Thoracic Surgery, 12, 706-712. https://doi.org/10.1016/S1010-7940(97)00253-4

[7] Staples, C.A., Muller, N.L., Miller, R.R., et al. (1988) Mediastinal Nodes in Bronchogenic Carcinoma: Comparison between CT and Mediastinoscopy. Radiology, 167, 367-372. https://doi.org/10.1148/radiology.167.2.3357944

[8] Dillemans, B., Deneffe, G., Verschakelen, J. and Decramer, M. (1994) Value of 
Computed Tomography and Mediastinoscopy in Preoperative Evaluation of Mediastinal Nodes in Non-Small Cell Lung Cancer. European Journal of Cardio-Thoracic Surgery, 8, 37-42. https://doi.org/10.1016/1010-7940(94)90131-7

[9] Rodriguez, P., Santana, N., Gamez, P. and Rodriguez De Castro, F. (2003) Mediastinoscopy in Diagnosis of Mediastinal Disease, An Analysis of 181 Explorations. Arch. Bronchoneumal., 39, 29-34.

[10] Yasufuku, K., Pierre, A., Darling, G., de Perrot, M., Waddell, T., Johnston, M., et al. (2011) A Prospective Controlled Trial of Endobronchial Ultrasound-Guided Transbronchial Needle Aspiration Compared with Mediastinoscopy for Mediastinal lymph Node Staging of Lung Cancer. The Journal of Thoracic and Cardiovascular Surgery, 142, 1393-1400. https://doi.org/10.1016/j.jtcvs.2011.08.037

[11] Steinert, H.C., Hauser, M., Allemann, F., et al. (1997) Non-Small Cell Lung Cancer: Nodal Staging with FDG-PET versus CT with Correlative Lymph Node Mapping and Sampling. Radiology, 202, 441-446. https://doi.org/10.1148/radiology.202.2.9015071

[12] Sasaki, M., Ichiya, Y., Kuwabara, Y., et al. (1996) The Usefulness of FDG Positron Emission Tomography for the Detection of Mediastinal Lymph Node Metastases in Patients with Non-Small Cell Lung Cancer: A Comparative Study with X-Ray Computed Tomography. European Journal of Nuclear Medicine, 23, 741-747. https://doi.org/10.1007/BF00843701

[13] de Langen, A.J., Raijmakers, P., Riphagen, I., Paul, M.A. and Hoekstra, O.S. (2006) The Size of Mediastinal Lymph Nodes and Its Relation with Metastatic Involvement: A Meta-Analysis. European Journal of Cardio-Thoracic Surgery, 29, 26-29. https://doi.org/10.1016/j.ejcts.2005.10.002

[14] De Leyn, P., Stroobants, S., De Wever, W., Lerut, T., Coosemans, W., Decker, G., et al. (2006) Prospective Comparative Study of Integrated Positron Emission Tomography-Computed Tomography Scan Compared with Remediastinoscopy in the Assessment of Residual Mediastinal Lymph Node Disease after Induction Chemotherapy for Mediastinoscopy-Proven Stage IIIA-N2 Non-Small-Cell Lung Cancer: A Leuven Lung Cancer Group Study. Journal of Clinical Oncology, 24, 3333-3339. https://doi.org/10.1200/JCO.2006.05.6341

[15] Metin, M., Citak, N., Sayar, A., Pekcolaklar, A., Melek, H., Kok, A., et al. (2011) The Role of Extended Cervical Mediastinoscopy in Stagin of Non-Small Cell Lung Cancer of the Left Lung and a Comparison with Integrated Positron Emission Tomography and Computed Tomography: Does Integrated Positron Emission Tomograpy and Computed Tomography Reduce the Need for Invasive Procedures? Journal of Thoracic Oncology, 6, 1713-1719. https://doi.org/10.1097/JTO.0b013e318225914e

[16] De Leyn, P., Schoonooghe, P., Deneffe, G., Van Raemdonck, D., Coosemans, W., Vansteenkiste, J. and Lerut, T. (1996) Surgery for Non-Small Cell Lung Cancer with Unsuspected Metastasis to Ipsilateral Mediastinal or Subcarinal Nodes (N2 Disease). European Journal of Cardio-Thoracic Surgery, 10, 649-655. https://doi.org/10.1016/S1010-7940(96)80380-0

[17] Cybulski, I.J., Lanza, L.A., Ryan, B., Putnam, J.B., McMurtrey, M.M. and Roth, J.A. (1992) Prognostic Significance of Computed Tomography in Resected N2 Lung Cancer. The Annals of Thoracic Surgery, 54, 533-537. https://doi.org/10.1016/0003-4975(92)90449-E

[18] De Leyn, P., Lardinois, D., Van Schil, P.E., Rami-Porta, R., Passlick, B., Zielinski, M, et al. (2007) ESTS Guidelines for Preoperative Lymph Node Staging for Non-Small 
Cell Lung Cancer. European Journal of Cardio-Thoracic Surgery, 32, 1-8. https://doi.org/10.1016/j.ejcts.2007.01.075

[19] Puhakka, H.J. (1989) Complications of Mediastinoscopy. The Journal of Laryngology \& Otology, 103, 312-315. https://doi.org/10.1017/S0022215100108795 